\title{
Editorial
}

\section{Artificial Intelligence as a Tool to Advance in Urology}

\author{
Herney Andrés García-Perdomo ${ }^{10}$ \\ ${ }^{1}$ Department of Surgery/Urology, School of Medicine, Universidad \\ del Valle, Cali, Colombia \\ Urol Colomb 2020;29:3-4.
}

As clinicians, surgeons, researchers, and academics, we face different challenges in the settings we act. We all know the complicated process of decision-making and the importance of preventing judgment errors in each step: When defining a diagnosis, addressing modifiable risk factors, optimizing resource use, offering or conducting surgery, and managing complications. ${ }^{1}$

Currently, the high volume of data, its availability, and the different existing sources might prevent the appropriate information synthesis to obtain an accurate diagnosis, plan an individualized treatment and get the prediction of an outcome. ${ }^{2}$ We are commonly confident in statistical methods, which are the usual tools that we see in published studies. Nevertheless, clinicians and researchers have applied artificial intelligence (AI) methods in different knowledge areas, specifically in urologic diseases for years outperforming the standard methods $s^{3,4}$ to overcome the problem we face every day in our decision-making process.

$\mathrm{AI}$ is a critical tool that focuses on creating an intelligent algorithm that performs human tasks by complex non-linear mathematical models and building blocks that mimic human neurons. The primary objective is learning easy and challenging human capabilities to improve a determined outcome. ${ }^{1,2}$ Therefore, different kinds of AI reflect computer systems that can learn from raw data with a degree of autonomy, for example, machine learning, artificial networks, natural learning process, deep learning, and reinforcement learning, among others. ${ }^{5}$

Machine learning (ML) is a subtype of AI in which there are science and application of statistical models and algorithms that can infer patterns from raw. This method was one the first of this kind and demonstrated that there was no need to write long codes, but machines could be taught and learn how to detect a pattern. Some examples include voice recognition, web search engines, and email filters. ${ }^{2}$ There are two models, supervised and unsupervised. The first one refers to systems that can infer a prescribed outcome or an output category from raw data by some instructions from human experts, in other words, the system must be trained to learn an association between the input data and the output (outcome). The unsupervised model creates its output categories from the structure and distribution of the input data without specific training. ${ }^{1}$

Another interesting type of AI is artificial neuronal networks (ANN) and deep learning (DL). They resemble a biological nervous system where signals are in layers of simple computational units (neurons); the connections among the units are weighted but can change as the network learns different input/output patterns. ${ }^{6}$ Deep-learning network is a highly-dimensional artificial neuronal network with multiple layers that support the discovery of hidden and abstract representations in raw data. Those hidden nodes and layers are weighted and are influenced by the previous layers as it learns. ${ }^{1,2}$

On the other side, reinforcement learning is another type of $\mathrm{AI}$ in which computer systems can identify actions that yield the highest probability of a given outcome. In this subtype, the model can be trained by trial and error scenarios (algorithms) to find these actions. ${ }^{1}$

The natural language processing comprehends a system that can understand human written and spoken language. This type of AI is crucial for the analysis of large-scale electronic medical records, especially for physicians' narrative data (clinical histories). It will identify standard information to find relationships. ${ }^{2}$

So far, I have described different subtypes of AI, but from here, I will describe some examples of the clinical applications in urology.

Most of the applications are described in genitourinary cancer regarding diagnosis, staging, and prognosis. Additionally, according to new advances in omics sciences, researchers have also put their attention in novel biomarkers and data mining by bioinformatics techniques (computational technologies and statistics for the biological data analysis) that also uses AI tools.

In prostate cancer, there are ML and ANNs that have shown improvement in diagnostic accuracy in some scenarios, especially in those with digital pathology and Magnetic Resonance (MR) of the prostate. However, in others such as staging and

Copyright ( $\odot$ 2020, Sociedad Colombiana License terms de Urología. Publicado por Thieme Revinter Publicações Ltda., Rio de Janeiro, Brazil. Todos los derechos reservados.
Address for correspondence Herney Andrés García-Perdomo, MD MSc EdD, PhD, FACS,

DOI https://doi.org/

10.1055/s-0040-1709122.

ISSN 0120-789X.

Department of Surgery/Urology, e ISSN 2027-0119.

School of Medicine, Universidad

del Valle, Cali, Colombia

(e-mail: editorrevista@scu.org.co). 
prognosis, some AI models demonstrated superiority over standard statistical tools, while others found similar results. ${ }^{2,6}$

Regarding urothelial cancer, the use of imaging and urine metabolite markers based on AI models might improve the diagnosis of bladder and urothelial cancer (AUC 0.7 to 0.9 ). It will also be essential to diminish the interobserver variability. Additionally, it may improve the prognosis in patients with the muscle-invasive disease. ${ }^{2,7}$

Renal cancer can be challenging, especially during the diagnosis process. Clinical and metabolomic databased on spectroscopy and mass spectrometry might be used in AI tools and improve the diagnostic accuracy over statistical or imaging conventional methods. ${ }^{2,8}$

Other settings in urology are hydronephrosis and urinary reflux. There are studies which try to predict urinary reflux and renal obstruction from ultrasonography based on ML. The authors found that they are slightly better than standard statistical tools. ${ }^{9,10}$

$\mathrm{AI}$ is an exciting and essential tool to support clinical decision-making for urologists. There are no enough data right now to standardize it; nevertheless, this is a tool that showed up to remain. We need to work together with the data scientist, the biostatistician, the molecular biologist, and other professionals that can collaborate to train these methods to improve our patients' health.

\section{Conflict of Interests}

The authors have no conflict of interests to declare.

\section{References}

1 Loftus T, Tighe P, Filiberto A, et al. Artificial intelligence and surgical decision-making. JAMA Surg 2019

2 Chen J, Remulla D, Nguyen JH, et al. Current status of artificial intelligence applications in urology and their potential to influence clinical practice. BJU Int 2019;124(04):567-577

3 Drouin SJ, Yates DR, Hupertan V, Cussenot O, Rouprêt M. A systematic review of the tools available for predicting survival and managing patients with urothelial carcinomas of the bladder and of the upper tract in a curative setting. World J Urol 2013;31 (01):109-116

$4 \mathrm{Kim} \mathrm{JK}$, Yook IH, Choi MJ, et al. A performance comparison on the machine learning classifiers in predictive pathology staging of prostate cancer. Stud Health Technol Inform 2017;245:1273

5 Hashimoto DA, Rosman G, Rus D, Meireles OR. Artificial intelligence in surgery: promises and perils. Ann Surg 2018;268(01): 70-76

6 Abbod MF, Catto JWF, Linkens DA, Hamdy FC. Application of artificial intelligence to the management of urological cancer. J Urol 2007;178 (4 Pt 1):1150-1156

7 Xu X, Zhang X, Tian Q et al. Three-dimensional texture features from intensity and high-order derivative maps for the discrimination between bladder tumors and wall tissues via MRI. Int J CARS 2017;12(04):645-656

8 Haifler M, Pence I, Sun Y, et al. Discrimination of malignant and normal kidney tissue with short wave infrared dispersive Raman spectroscopy. J Biophotonics 2018;11(06):e201700188

9 Logvinenko T, Chow JS, Nelson CP. Predictive value of specific ultrasound findings when used as a screening test for abnormalities on VCUG. J Pediatr Urol 2015;11(04):176.e1-176.e7

10 Cerrolaza JJ, Peters CA, Martin AD, Myers E, Safdar N, Linguraru MG. Quantitative ultrasound for measuring obstructive severity in children with hydronephrosis. J Urol 2016;195(4 Pt 1):1093-1099 